\title{
RANKing the importance of measles virus in Paget's disease
}



\section{F. Patrick Ross}

Department of Pathology, Washington University School of Medicine, Barnes-Jewish Hospital North, Mail Stop 90-31-649, 216 South Kingshighway, St. Louis, Missouri 63110, USA.

Phone: (314) 454-8079; Fax: (314) 454-5505; E-mail: rossf@medicine.wustl.edu.

Paget's disease, a common, multifocal skeletal disorder, provides a paradigm of bone remodeling gone awry. Both bone resorption and formation occur at an increased pace in pagetic bone, but the pathology arises in osteoclasts, cells that continuously migrate through and degrade the mineralized extracellular matrix of the bone. Reflecting its increased resorptive activity, the pagetic osteoclast is large and hypernucleated. Early clues regarding pathogenesis came with recognition that the nuclei and cytosol of pagetic osteoclasts contain inclusions reminiscent of structures seen in virus-infected cells. Subsequent studies demonstrated mRNA for measles, respiratory syncytial, or canine distemper virus - all members of the paramyxovirus family - in the osteoclasts of a majority of pagetic patients (1). A recent report that erythroid cells from patients with Paget's disease express viral mRNA (2), along with the delayed and unpredictable expression of the phenotype, suggests that some generally quiescent hematopoietic stem cell serves as the primary host for viral infection. However, the presence of viral mRNA in mesenchymal cells in bone (3) raises the possibility that nonhematopoietic cells also play a role in the disease.

Reddy et al. have documented 2 major alterations in the milieu in pagetic bone that are likely to have mechanistic implications. First, unlike their normal counterparts, pagetic osteoclasts and their precursors secrete high levels of IL-6. Second, bone marrow aspirates from patients are hypersensitive to the effects of 1,25 dihydroxyvitamin $\mathrm{D}_{3}\left(1,25(\mathrm{OH})_{2} \mathrm{D}_{3}\right)$ and differentiate to form osteoclast-like cells

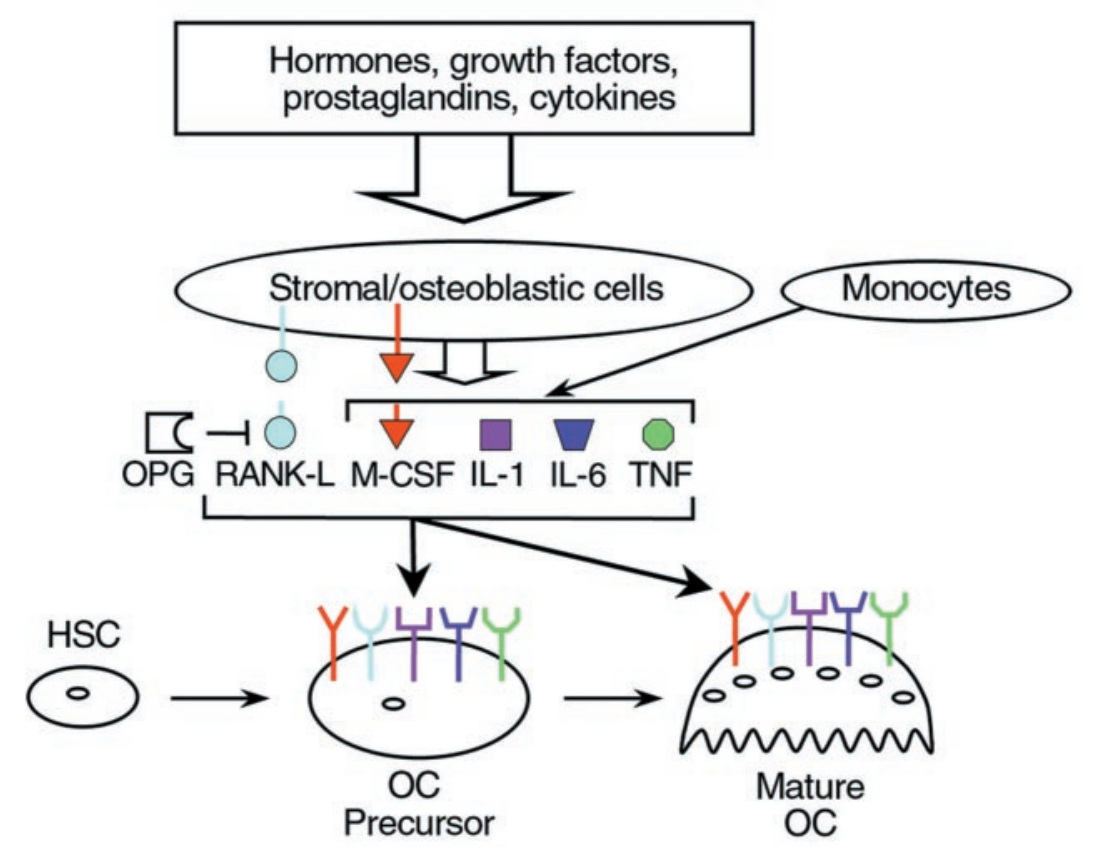

Figure 1

Regulation of osteoclast formation. A range of hormones and cytokines, targeting to stromal cells, enhance expression of M-CSF and RANK-L, the pivotal osteoclastogenic molecules, and the proinflammatory cytokines IL-1, IL-6, and TNF. These proteins stimulate both formation and activity of mature polykaryons. HSC, hematopoietic stem cell; OC, osteoclast.

when treated with doses of this compound 10 -fold lower than needed to induce this transition in healthy cells (4). The presence of viral mRNA in osteoclasts first suggested that 1 or more viral proteins prime cells to undergo these phenotypic changes, and the report by Kurihara et al. in this issue of the JCI (5) confirms that expression of measles virus nucleocapsid protein (MVNP) in normal, marrow-derived $\mathrm{CD} 4^{+}$cells promotes increased bone resorption, enhances secretion of IL- 6 by osteoclasts, and increases their sensitivity to $1,25(\mathrm{OH})_{2} \mathrm{D}_{3}$. Introduction of the measles virus membrane (MVM) protein causes different responses, suggesting that the latter protein plays a distinct role in inducing deranged bone turnover. In addition, the authors document that osteoclast precursors transduced with either MVNP- or MVM-enhanced activation of the NFKB transcription factor complex, a central aspect of osteoclast biology.

As discussed below, and of considerable interest, these findings suggest that the causative agents of Paget's disease circumvent the currently accepted pathway for osteoclast formation and activation (summarized in Figure 1). The key elements in this pathway are the receptor for activation of NFKB (RANK), which is found on osteoclasts and their precursors; the RANK ligand (RANK-L; also referred to in the literature as TRANCE, OPGL, and ODF), produced in both soluble and membrane-bound forms by osteoblasts and stromal cells in bone marrow; osteoprotegerin (OPG), a soluble product of osteoblasts and stromal cells, which acts as a decoy receptor for RANK-L; and macrophage colony-stimulating factor (M-CSF), a product of stromal cells or monocytes, which binds to the receptor $c$-fms on osteoclasts to promote their differentiation, survival, and proliferation (6). As predicted by this model, purified murine or human monocytes and macrophages treated 


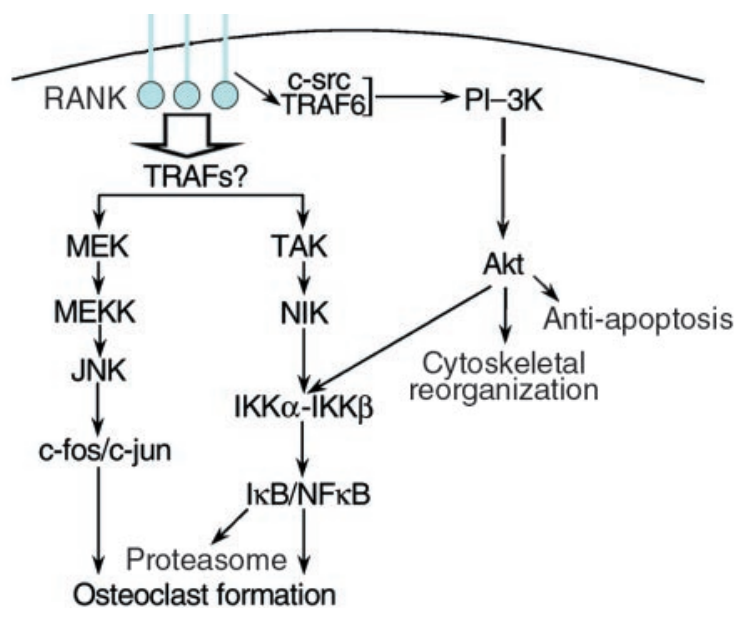

Figure 2

Key elements of RANK signaling. Receptor ligation is followed by recruitment of adaptor molecules, including TRAF6, which, interacting with c-src, stimulates the PI-3 K/Akt pathway. The TRAF proteins that activate MEK and TAK have not been identified conclusively, but these kinases initiate signaling cascades that activate AP-1 transcription factors (consisting of c-fFos and c-jun) and promote the proteasomal degradation of the NFKB inhibitor IKB. Akt plays a role in phosphorylation of the IKK complex (25). AP-1 and NFKB serve as key activators of osteoclast formation and function.

with M-CSF and RANK-L give rise to numerous functional osteoclasts (7), and this process can be blocked in a dose-dependent manner by OPG (8). In addition, injection of RANK-L into mice results in a rapid $(<1$ day) increase in serum calcium, indicating that the cytokine activates mature osteoclasts, which accelerate the resorption of bone (9). The proinflammatory cytokines IL-1, TNF $\alpha$, and IL6 , the products of stromal cells and monocytes, not only regulate osteoclastogenesis by stromal cells, but also act directly on osteoclasts and their precursors to promote cell fusion and altered function and survival $(6,10$, 11). Finally, the phenotypes of mice carrying targeted deletion of the RANK$L, O P G$, and RANK genes support the current model. Mice lacking either RANK-L or RANK are deficient in osteoclasts and, hence, are severely osteopetrotic. The absence of OPG, conversely, results in profound, accelerated osteoporosis (reviewed in ref. 8).

Signaling by RANK and its ligand. Given that RANK-L is the critical cytokine stimulating differentiation and fusion of precursors into mature osteoclasts, the nature of the intracellular signals that follow RANK activation is of considerable interest. The known proximal and distal signaling events arising from ligation of RANK (Figure 2) are similar to those of other members of the TNF receptor superfamily. Two critical distal events in RANK signaling are activation of the NFKB complex and the transcription factor AP-1, a dimer of the cfos and c-jun proteins. Again, gene-targeting studies confirm the significance of each of these gene products for osteoclast biology. Mice lacking both p50 and p52, members of the NFKB family, exhibit profound osteopetrosis (12). Similarly, animals lacking c-fos generate no multinucleated, boneresorbing cells (13), and precursor cells that over-express a mutated form of cjun that cannot be activated by the kinase JNK are also blocked in this differentiation pathway (14).

Understanding the proximal signals initiated by RANK activation has proved more complex. Overexpression of RANK leads to binding of 5 members of the TRAF family $(1-3,5,6)$, proteins that link the receptor to numerous downstream signals (15), including cytoskeletal reorganization, antiapoptotic signals, and activation of NFKB, all key aspects of osteoclast function. Which of these adaptors mediate RANK signaling in normal tissue is uncertain. Binding of TRAF1 to RANK has been shown only under conditions of overexpression (15) and thus may not be physiologically relevant. Mice lacking TRAF 2, 3, or 5 exhibit a range of immunological defects but have normal bones (ref. 16 and references therein), possibly indicating that these TRAFs have redundant roles in the bone. The double or triple knockouts needed to address their common function in this tissue have not yet been described. On the other hand, the absence of TRAF 6 results in osteopetrosis in the face of numerous nonfunctional osteoclasts (17), a phenotype reminiscent of the c-src knockout (18). Indeed, following receptor ligation, c-src interacts with TRAF6 (19) and activates downstream signaling pathways, which are evidently dispensable for osteoclastogenesis but required to stimulate bone resorption. In addition, poorly characterized TRAF-independent signals may arise from the cytoplasmic tail of the activated RANK. In murine osteoclasts, RANK-derived signals initiate a cascade of kinases (19), including MEK kinase, MEK, and JNK1 (S. Srivastava, M.N. Weitzmann, F.P. Ross, and R. Pacifici, unpublished data).

Physiological regulation of bone resorption. Work published over the last year reveals that many humoral or local factors that modulate osteoclast function do so, wholly or in part, by altering expression of M-CSF, OPG, or RANK-L. These findings, summarized in Table 1 , must be considered in the light of a model in which the biological efficacy of RANK-L relative to OPG determines the net outcome. Factors that increase RANK-L expression or suppress OPG increase osteoclastogenesis, whereas the converse changes diminish it. When both regulatory proteins are altered in the same direction the relative magnitude of the changes determines whether osteoclast number and activity rise or fall. Thus, prostaglandins and parathyroid hormone $(\mathrm{PTH})$, which increase RANK-L while decreasing OPG, promote bone resorption. Similarly, TGF$\beta$, which diminishes RANK- $L$ and enhances OPG expression, decreases osteoclast activity. Estrogen, by dampening M-CSF and stimulating OPG, blunts 2 distinct pathways necessary for osteoclast formation and survival. In the cases of IL-6, IL-11, and BMP-2, each of which modulates only OPG or RANK-L, there is concordance between the direction of the changes and the known biological activity of each molecule, namely enhanced resorption.

This analysis is more complex in the case of the IL-1, TNF $\alpha$, and $1,25(\mathrm{OH})_{2} \mathrm{D}_{3}$, all potent stimulators of bone resorption. Whereas these $3 \mathrm{~mol}-$ ecules each increase production of both RANK-L and OPG, the dominant 
outcome in all 3 cases is a net increase in RANK-L activity. Complicating matters further, IL-1, IL-6, and TNF $\alpha$ control their own expression and that of RANK-L, OPG, and/or M-CSF; but, as mentioned, they may also act directly on osteoclast precursors and the mature bone-resorbing cell. In vivo, the rate of osteoclastogenesis responds to the net sum of all signals activating and inhibitory signals, and the entire process is subject to ongoing and rapid modulation.

$R A N K-L$-independent induction of pagetic osteoclasts? The emergence of the pagetic phenotype - numerous, large osteoclasts with markedly enhanced bone resorption - in virally infected cells expressing may be explained largely by invoking higher local levels of IL-6 and greater target cell sensitivity to $1,25(\mathrm{OH})_{2} \mathrm{D}_{3}$, phenomena described previously by the authors of the current report. Based on the model in Figure 1, raising IL-6 production is expected to promote RANK-L expression and, hence, increase osteoclast number and activity. Alternatively, since MNVP-transduced cells express higher levels of RANK, and cells containing MVM can be induced to do so upon exposure to $1,25(\mathrm{OH})_{2} \mathrm{D}_{3}$, it is possible that higher RANK levels on osteoclast precursors also contribute to the pagetic phenotype. Pagetic cells are unusually large, often containing more than 100 nuclei. The reason for this feature is less clear, but it may result from the action of IL-1, which is induced by a paracrine effect of IL- 6 on stromal cells or monocytes and which stimulates fusion of osteoclast precursors (6).

\section{Table 1}

Summary of the factors that modulate expression of RANK-L, OPG, and M-CSFA

\begin{tabular}{|c|c|c|c|}
\hline Hormones & RANK-L & OPG & $\mathrm{M}-\mathrm{CSI}$ \\
\hline $1,25(\mathrm{OH})_{2}$ vitamin $\mathrm{D}_{3}$ & $\uparrow$ & $\uparrow$ & $\uparrow$ \\
\hline Estrogen & & $\uparrow$ & $\downarrow$ \\
\hline $\mathrm{PTH}^{\mathrm{B}}$ & $\uparrow$ & $\downarrow$ & \\
\hline \multicolumn{4}{|l|}{ Cytokines } \\
\hline TNF- $\alpha$ & $\uparrow$ & $\uparrow$ & $\uparrow$ \\
\hline IL-1 & $\uparrow$ & $\uparrow$ & \\
\hline IL-6 & $\uparrow$ & & \\
\hline IL-11 & $\uparrow$ & & \\
\hline \multicolumn{4}{|l|}{ Growth Factors } \\
\hline TGF- $\beta$ & $\downarrow$ & $\uparrow$ & \\
\hline BMP-2 & & $\uparrow$ & \\
\hline \multicolumn{4}{|l|}{ Others } \\
\hline Prostaglandin $E_{2}$ & $\uparrow$ & $\downarrow$ & \\
\hline
\end{tabular}

All relevant in vitro studies performed by the San Antonio group $(4,5)$ used CD34 ${ }^{+}$myeloid cells exclusively, which are presumed to make no RANK-L. Because RANK-L is normally a regulator sine qua non of osteoclast formation and function, this cytokine would thus appear to be peripheral to the pagetic phenotype of paramyxovirus-infected cells. If so, one must deduce that the virus, acting intracellularly in osteoclast precursors, can trigger signaling by unligated RANK. It should be noted, however, that a subset of marrowderived human $\mathrm{CD} 4^{+}$cells also stain for the stromal-specific marker STRO (20), so the culture may contain stromal cells that secrete RANK-L. This possibility could be addressed by studying stroma-free cultures that express MVNP or by blocking RANK-L. If stromal elements prove not to be required for the pagetic response of cultured virus-infected osteoclasts, these studies will confirm the existence of RANK$\mathrm{L}$-independent signaling in precursors. This possibility is suggested by the current finding that, whereas treatment of control cells with RANK-L causes only a modest increase in the amount of $\mathrm{NF \kappa B}$ binding to a consensus response element, this complex is maximally activated in both untreated MVNP-expressing cells and in MVM-expressing cells treated with RANK-L. Taken together, these observations indicate that one, and perhaps more, viral proteins augment RANK signaling, with MVNP doing so in a ligand-independent manner. Furthermore, because $1,25(\mathrm{OH})_{2} \mathrm{D}_{3}$ enhances RANK expression in MVM- transduced cells, higher levels of surface-expressed RANK may interact with stroma-derived RANK-L to enhance osteoclast formation and activity.

A very recent report linking osteoclast dysfunction with alterations in RANK signaling (21) provides important insights into a potentially novel mechanism that may be involved in Paget's disease. Patients with familial expansile osteolysis, a rare inherited condition that, like Paget's disease, is manifested with increased bone resorption, bear an in-frame insertion within the first exon of the RANK gene. This mutation interferes with cleavage of RANK's signal peptide, and when the mutated gene was expressed in human embryonic renal cells, surface expression of the mutant protein reached only $25 \%$ of wild-type levels. Presumably, the uncleaved precursor accumulates intracellularly in a functional form. Supporting this hypothesis, a cotransfected NFKB reporter gene is active in these cells, even in the absence of RANK-L (21). Cell-culture studies also show that RANK overexpression can result in ligand-independent signal transduction (22).

Taken together with the enhanced expression of RANK on pagetic osteoclasts and their precursors, these findings raise the possibility that measles, and, by implication, other paramyxoviruses, can stimulate bone resorption by several mechanisms. First, there may be increased production of stromal-derived osteoclastogenic cytokines (stimulated by IL- 6 secreted by infected osteoclasts and their precursors). Second, it is possible that both RANK-L-independent and-dependent myeloid signaling are stimulated, with the latter influenced by raised levels of intracellularand/or surface-residing RANK, secondary to viral infection and increased sensitivity to $1,25(\mathrm{OH})_{2} \mathrm{D}_{3}$.

A separate question, not addressed in the current report, relates to the fact that $\mathrm{CD} 4^{+}$cells infected with either MVNP or MVM do not require M-CSF for osteoclast formation. Once again, it can be hypothesized that stromal contamination provides the cytokine. Alternatively, the finding that the macrophage-like line RAW 264.7 gives rise to osteoclasts in the absence of exogenous M-CSF (23) suggests the possibility of autonomous activation of c-fms signaling. Whereas an activating 
mutation in the c-fms gene has been described (24), the structure of this gene in RAW cells in unknown.

In conclusion, the present report is important because it provides convincing evidence that paramyxoviral proteins can induce Paget's disease and provides hints as to the mechanism that drives the disease. Dissection of the molecular details will be challenging, but should enrich both virology and bone biology.

1. Singer, F.R. 1999. Update on the viral etiology of Paget's disease of bone. J. Bone Miner. Res. 14(Suppl.):29-33.

2. Reddy, S.V., et al. 1999. Measles virus nucleocapsid transcript expression is not restricted to the osteoclast lineage in patients with Paget's disease of bone. Exp. Hematol. 27:1528-1532.

3. Gordon, M.T., Anderson, D.C., and Sharpe, P.T 1991. Canine distemper virus localised in bone cells of patients with Paget's disease. Bone 12:195-201.

4. Reddy, S.V., Menaa, C., Singer, F.R., Demulder, A and Roodman, G.D. 1999. Cell biology of Paget's disease. J. Bone Miner. Res. 14(Suppl.):3-8.

5. Kurihara, N., Reddy, S.V., Menaa, C., Anderson, D., and Roodman, G.D. 2000. Osteoclasts formed by normal human bone marrow cells transduced with the measles virus nucleocapsid gene express a pagetic phenotype. J. Clin. Invest. 105:607-614 (2000).

6. Takahashi, N., Udagawa, N., and Suda, T. 1999. A new member of tumor necrosis factor ligand family, ODF/OPGL/TRANCE/RANKL, regulate osteoclast differentiation and function. Biochem. Biophys. Res. Commun. 256:449-455.
7. Quinn, J.M., Elliott, J., Gillespie, M.T., and Martin, T.J. 1998. A combination of osteoclast differentiation factor and macrophage-colony stimulating factor is sufficient for both human and mouse osteoclast formation in vitro. Endocrinology. 139:4424-4427.

8. Hofbauer, L.C., et al. 2000. The roles of osteoprotegerin and osteoprotegerin ligand in the paracrine regulation of bone resorption. J. Bone Miner. Res. 15:2-12.

9. Burgess, T.L., et al. 1999. The ligand for osteoprotegerin (OPGL) directly activates mature osteoclasts. J. Cell Biol. 145:527-538.

10. Adebanjo, O.A., et al. 1998. Mode of action of interleukin- 6 on mature osteoclasts. Novel interactions with extracellular $\mathrm{Ca} 2+$ sensing in the regulation of osteoclastic bone resorption. J. Cell Biol. 142:1347-1356.

11. Kobayashi, K., et al. 2000. Tumor necrosis factor $\alpha$ stimulates osteoclast differentiation by a mechanism independent of the ODF/RANKL-RANK interaction. J. Exp. Med. 191:275-285.

12. Franzoso, G., et al. 1997. Requirement for NFkappaB in osteoclast and B-cell development. Genes Dev. 11:3482-3496.

13. Grigoriadis, A.E., et al. 1994. c-Fos: a key regulator of osteoclast-macrophage lineage determination and bone remodeling. Science. 266:443-448.

14. David, J.-P., et al. 1999. c-Jun phosphorylation by JNK1 but not by JNK2 is necessary for efficient osteoclastogenesis in response to TRANCE stimulation. J.Bone Miner. Res. 14:S149 (Abstr.)

15. Arch, R.H., Gedrich, R.W., and Thompson, C.B 1998. Tumor necrosis factor receptor-associated factors (TRAFs) - a family of adapter proteins that regulates life and death. Genes Dev. 12:2821-2830

16. Nakano, H., et al. 1999. Targeted disruption of Traf5 gene causes defects in CD40- and CD27mediated lymphocyte activation. Proc. Natl. Acad. Sci. USA. 96:9803-9808.
17. Lomaga, M.A., et al. 1999. TRAF6 deficiency results in osteopetrosis and defective interleukin-1, CD40, and LPS signaling. Genes Dev. 13:1015-1024

18. Soriano, P., Montgomery, C., Geske, R., and Bradley, A. 1991. Targeted disruption of the c-src proto-oncogene leads to osteopetrosis in mice. Cell. 64:693-702.

19. Wong, B.R., et al. 1999. TRANCE, a TNF family member, activates Akt/PKB through a signaling complex involving TRAF6 and c-Src. Molecular Cell. 4:1041-1049.

20. Simmons, P.J., and Torok-Storb, B. 1991. CD34 expression by stromal precursors in normal human adult bone marrow. Blood. 78:2848-2853.

21. Hughes, A.E., et al. 2000. Mutations in TNFRSF11A, affecting the signal peptide of RANK, cause familial expansile osteolysis. Nat. Gen. 24:45-48.

22. Wong, B.R., et al. 1998. The TRAF family of signal transducers mediates NF-KAPPA-B activation by the TRANCE receptor. J. Biol. Chem 273:28355-28359.

23. Hsu, H., et al. 1999. Tumor necrosis factor receptor family member RANK mediates osteoclast differentiation and activation induced by osteoprotegerin ligand. Proc. Natl. Acad. Sci. USA. 96:3540-3545.

24. Roussel, M.F., Downing, J.R., Rettenmier, C.W. and Sherr, C.J. 1988. A point mutation in the extracellular domain of the human CSF-1 receptor (c-fms proto-oncogene product) activates its transforming potential. Cell. 55:979-988.

25. Ozes, O.N., et al. 1999. NF-kappaB activation by tumour necrosis factor requires the Akt serine-threonine kinase [see comments]. Nature. 401:82-85

26. Lee, S.K., and Lorenzo, J.A. 1999. Parathyroid hormone stimulates TRANCE and inhibits osteoprotegerin messenger ribonucleic acid expression in murine bone marrow cultures: correlation with osteoclast-like cell formation. Endocrinology. 140:3552-3561. 